\title{
Proceso de rotura de una cúpula oval en la catedral de Tortosa. Análisis del mecanismo de colapso
}

\section{Cracking process of a oval dome in the cathedral of Tortosa. Assessment of the collapse mechanism}

L. Lluis i Ginovart( ${ }^{(*)}$, A. Costa ${ }^{(*)}$, G. Fortuny ${ }^{(*)}$, P. Solà-Morales ${ }^{(*)}$, J.M. Toldrà(*)

RESUMEN

En abril del 2011, una de las cúpulas tabicadas del recinto de la catedral de Tortosa (Tarragona), construida a finales del siglo XVIII, rompió repentinamente. El artículo presenta la evolución de la rotura y sus causas, analizando el progresivo proceso de fisuración iniciado con el descentramiento de la linterna. Con el objetivo de conocer el comportamiento de la cúpula y su capacidad portante, se analizan las condiciones de equilibrio a partir del estudio constructivo inicial. Se utilizan de manera complementaria métodos gráficos tradicionales y modelos tridimensionales por elementos finitos (MEF) con el programa libre Salome-Meca 6.3. A partir de la interpretación de los resultados, el estudio concluye que la cúpula por sí sola no tiene capacidad para mantenerse en equilibrio bajo el peso de la linterna. Así el efecto de las termitas sobre la sección de una de las vigas de cubierta provocará el asiento de la linterna y, en consecuencia, la formación de rótulas de colapso a partir de la distribución asimétrica de las cargas.

\section{SUMMARY}

In April of 2011, one of the masonry domes of the cathedral of Tortosa enclosure (Tarragona), built at late XVIII century, suddenly broke. The paper presents the evolution of the rupture and its causes, analyzing the cracking process initiated with the runout of the lantern. It is analyzed the equilibrium conditions starting from the initial constructive assessment in order to know the behavior of the dome and its bearing capacity. There are used complementary traditional graphical methods and tridimensional models by finite elements (FEM) by means of the free software Salome-Meca 6.3. Through the interpretation of the results, the assessment concludes that the dome by itself can hold the weight of the lantern. Thus, the cracking of one of the roof beams causes the bending of the lantern, and therefore the formation of collapse joints from the asymmetrical distribution of loads.

Keywords: Oval domes; masonry domes; analysis by FEM; limit Analysis.
Palabras clave: Cúpulas ovales; cúpulas de fábrica; análisis MEF; análisis límite.
$102-5$

(*) Universitat Rovira i Virgili. Reus (España).

Persona de contacto/Corresponding author: josep.lluisg@urv.cat (J. Lluis i Ginovart)
Recibido/Received: 19 jul 2012 Aceptado/Accepted: 25 nov 2012 
1. Planimetría general de la catedral y sección de la cúpula.

2. Esquema del entrevigado de cubierta y nomenclatura (vista cenital).

\section{LA CÚPULA OVAL Y SU ROTURA}

El ábside de la catedral de Tortosa fue construido entre los siglos XIV y XV (1). En 1773 se anexó a una de las capillas radiales el cuerpo que alberga la Sacristía Mayor, edificado por Antonio Ferrer. Consta de tres espacios diferenciados: el destinado a sacristía, presidido por una cúpula sexapartita, y dos colaterales, la capilla de la Reserva y el del Tesoro de la catedral. Se accede al cuerpo principal por un espacio intermedio entre el ábside y la Sacristía, cubierto por una cúpula oval con linterna, objeto del presente estudio, construida en 1784 por Josep Melet. Este espacio tamiza la iluminación entre la luz gótica del ábside y la neoclásica de la Sacristía.

La cúpula cubre un espacio de planta rectangular de dimensiones $460 \times 371 \mathrm{~cm}$, con la imposta situada a $833 \mathrm{~cm}$ desde el nivel del suelo (Figura 1). Se trata de una cúpula de planta oval que se desarrolla a partir de dos ejes perpendiculares de 455 y $365 \mathrm{~cm}$ respectivamente, y cuya cáscara es un ovoide de revolución.
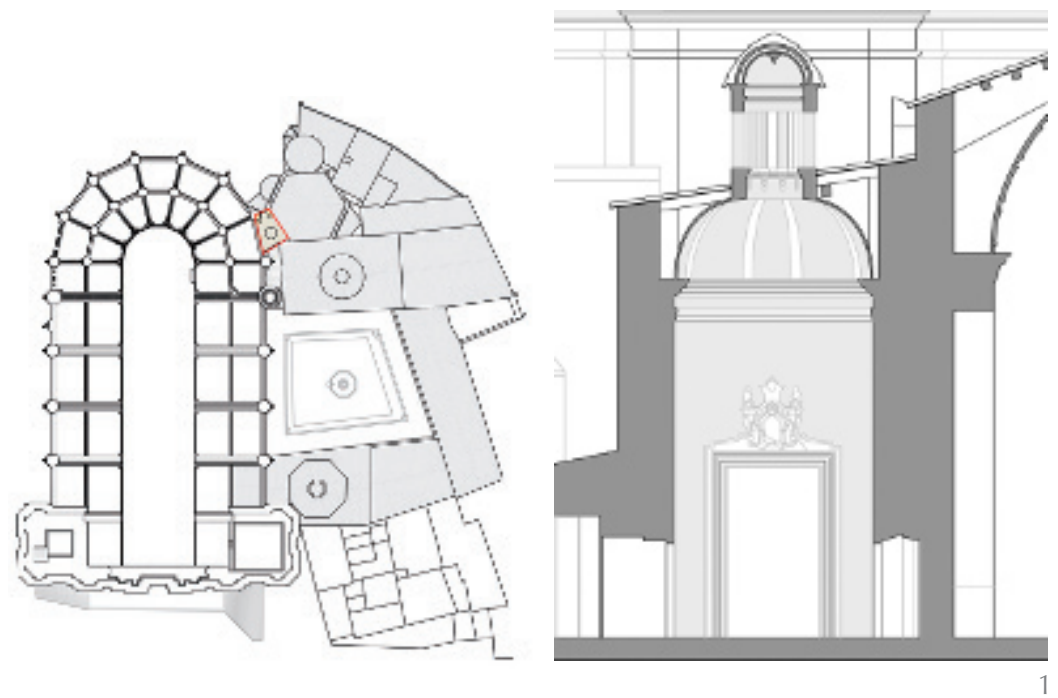

Dispone de un óculo ligeramente ovalado que se sitúa a una altura desde la imposta que oscila entre los $177 \mathrm{~cm}$ y $186 \mathrm{~cm}$, ya que la solución geométrica de la intersección con la cáscara tiene directriz curva. Sobre éste se encuentra la linterna, con una altura de $385 \mathrm{~cm}$ y rematada por un cupulín esférico de $146 \mathrm{~cm}$ de diámetro interior.

La cúpula arranca con una serie de hiladas paralelas al plano horizontal hasta alcanzar una altura de $30 \mathrm{~cm}$ desde la base del trasdós. A partir de este punto, la cáscara se construye con dos hojas de ladrillo manual de $35 \times 18 \times 3 \mathrm{~cm}$ aproximadamente, revocadas por ambas caras con mortero de yeso negro, alcanzando un grosor total de unos $9 \mathrm{~cm}$.

El casquete se refuerza con 8 nervios meridionales, doblándolo por ambas caras alcanzando un grosor de unos $17 \mathrm{~cm}$ (4 hojas de rasilla). El óculo se refuerza también por la cara interior con un grueso de rasilla formando un collarín de $18 \mathrm{~cm}$ de ancho, y por la cara exterior con un tambor de $30 \mathrm{~cm}$ de ancho sobre el que apoya la linterna. Ésta consta de ocho pilares de ladrillo manual y cerramiento de alabastro, que sustentan un cupulín de doble bóveda de hoja de rasilla con cámara intermedia y acabado de teja árabe.

Las vigas de madera que forman la cubierta tienen una disposición singular (Figura 2), ya que unas apoyan sobre los muros perimetrales y otras directamente sobre la cúpula. Aquellas situadas fuera de la proyección de la linterna atraviesan todo el espacio de cubierta y se empotran en los muros. Existen dos vigas tangentes al tambor, sobre las que apoya parte de la linterna, también empotradas en los muros, mientras que las vigas centrales apoyan directamente sobre el tambor. La cubrición se realizaba con rastreles de madera y teja árabe apoyados en el entrevigado formando un plano inclinado con una sola pendiente.
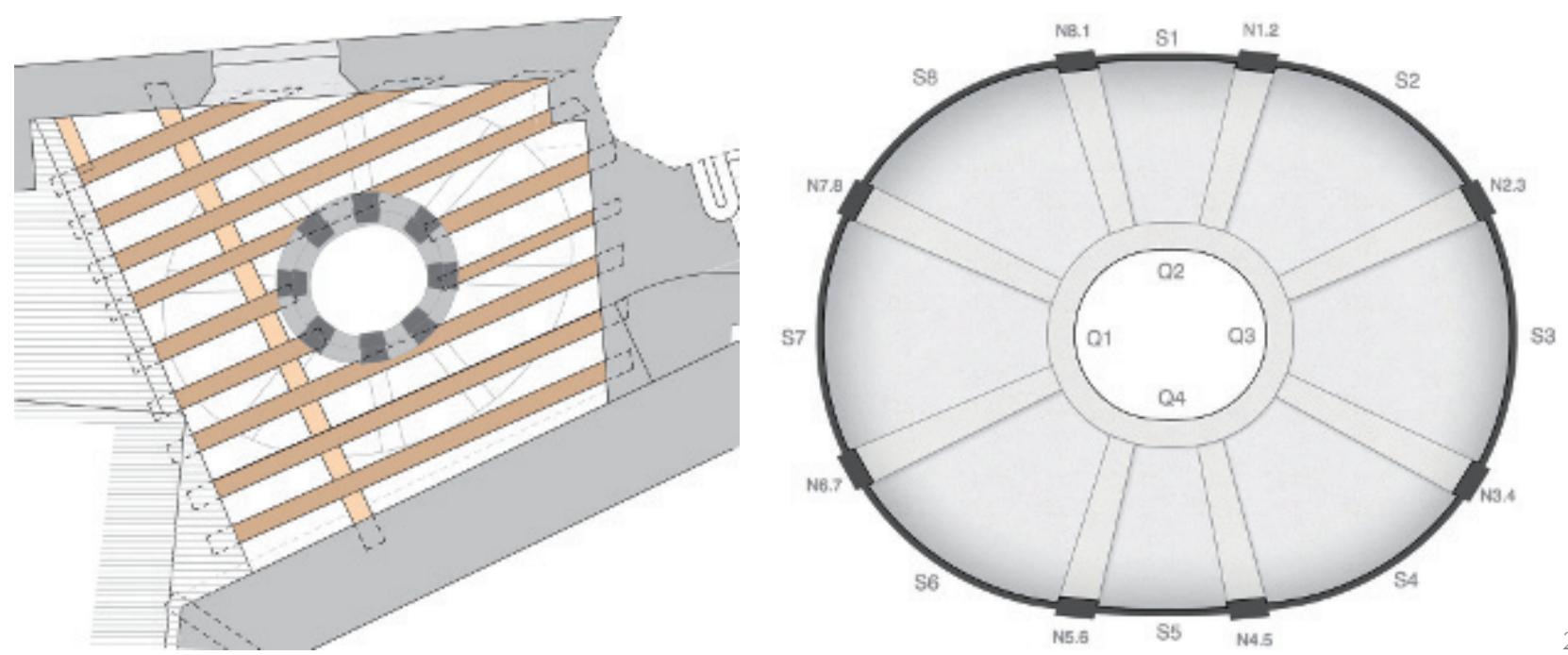

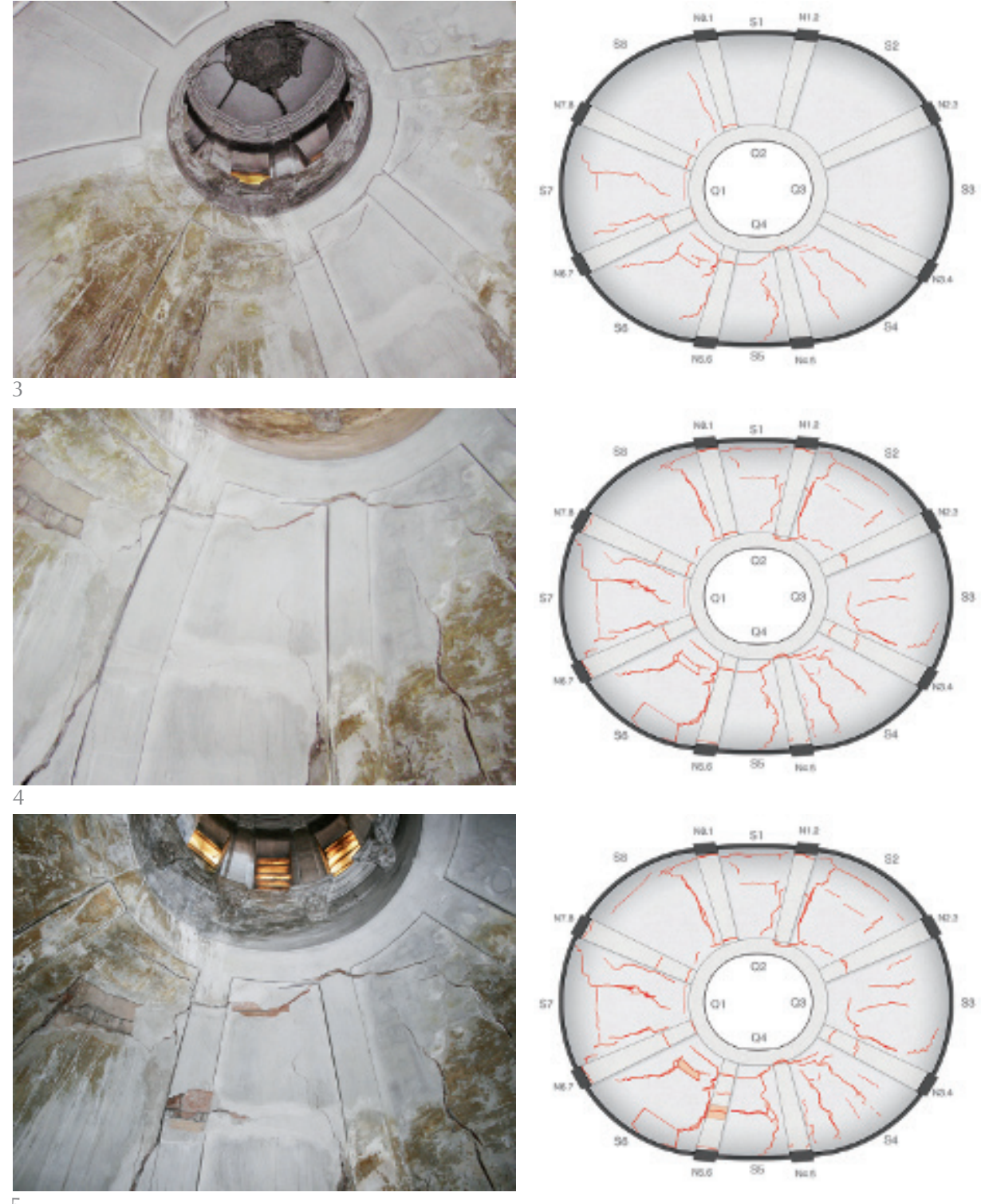

El trasdós de la cúpula estaba relleno de escombro con una altura muy variable de entre unos $45 \mathrm{~cm}$ y $100 \mathrm{~cm}$. Se encontraron restos de proyectiles probablemente originarios del bombardeo del ejército francés del 30 de diciembre de 1810.

La cúpula presentaba desde antaño diversas patologías, y debido a ello se realizó una visura el 12 de enero de 2011 (Figura 3), donde se determinó el desplome de la linterna en dirección Noroeste, en el nervio N5.6 entre las zonas S5 y S6. En la visura se apreciaron fisuras meridianas en S3, S4, S5, S6 y S7, S8 y paralelas alrededor del óculo en S4, S5, S6, S7 y S8, así como en los nervios N4.5, N5.6, N6.7, N7.8 y N8.1.

En la mañana del 26 de abril de 2011 (Figura 4), se aprecia la caída de material debido a la rotura horizontal en la parte superior de la cúpula, en los casquetes S5, S6 y S7 y de los nervios N5.6 y N6.7. Se observa la aparición de nuevas fisuras, tanto horizontales como verticales, así como la apertura de las ya existentes.

Ante la aparición de fisuras horizontales en la parte superior de la cúpula, se presagia el aumento del desplome de la linterna. La caída de material determina el inicio del mecanismo cinemático del conjunto cúpula y linterna, y en consecuencia se ordena el apuntalamiento y acodalamiento superior de la linterna sobre la cubierta del ábside de la catedral.

En la mañana del día 27 (Figura 5) se observa una nueva rotura en el nervio N5.6 y los casquetes S5 y S6. Paulatinamente se produce un aumento progresivo de la fisuración vertical y el inicio de un agrietamiento horizontal en varios casquetes, con la posible formación de tres articulaciones y por tanto del mecanismo de colapso de la cúpula.

En los días posteriores se dispuso una cubierta provisional con el fin de evitar
3. Visura cúpula 12 de enero de 2011.

4. Fisuración y caída de material 26 abril 2011.

5. Nueva fisuración y caída de material 27 abril 2011. 
6. Trasdós de la bóveda, 12 de mayo de 2011. filtraciones, realizando una cata bajo la cubierta de teja para inspeccionar el estado del trasdós de la cúpula. De la inspección se dedujo que la linterna se apoyaba directamente sobre la cúpula y sobre unas vigas de madera que formaban parte de la cubierta de teja árabe (Figura 6). La estructura de madera había sido objeto de la acción de las termitas, perdiendo sección resistente. Esto provocó el descenso del plano de cubierta y el desplome de la linterna.

Una vez determinado el origen mecánico de las lesiones, se deduce que las deformaciones de la cúpula están causadas por la aplicación de una carga asimétrica en el óculo, como consecuencia del proceso de acomodación de la fábrica a las nuevas solicitaciones. Conforme se deforma la cúpula, aparecen en primera instancia las grietas meridianas, seguidas de las rótulas (grietas paralelas), primero en la parte superior cercana al óculo, y en un segundo estadio en la parte inferior. Este proceso habría llevado al colapso de la estructura si no se hubiera detenido el mecanismo (2) (3).

\section{LAS DEFORMACIONES DE LA CÚPULA. FORMACIÓN DE ARTICULACIONES}

Una vez estabilizado el movimiento de la cúpula, se realizó un levantamiento fotogramétrico del intradós del casquete. Se concretó un modelo 3D con el programa Rhinoceros 3.0 a partir de la captura de 287 puntos para definir la geometría de los nervios, y 38.297 puntos para determinar la cáscara (Figura 7).

A partir del levantamiento se analizaron las deformaciones sufridas por cáscara y nervios tomando como referencia las secciones menos deformadas (Figuras 8 y 9 ). Los resultados determinaron que las deformaciones más importantes se habían producido en el casquete $\mathrm{S} 6$, con un descenso en el óculo del orden de $8 \mathrm{~cm}$.

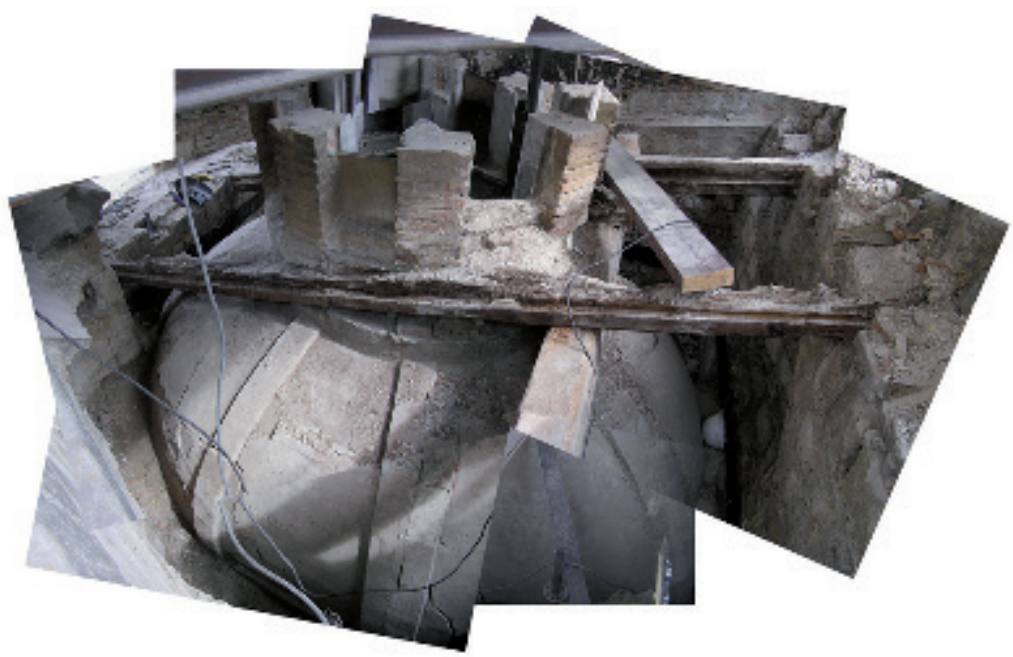

Los casquetes S3, S4 y S6 se deformaron del mismo modo aunque con diferentes valores; $3 \mathrm{~cm}, 3,5 \mathrm{~cm}$ y $7 \mathrm{~cm}$ respectivamente. El resto de casquetes se deformaron con un desplazamiento horizontal hacia el exterior de la sección inferior, acompañado de un descenso de la mitad superior. En este caso, el casquete más deformado es el S5, con desplazamientos de hasta $6 \mathrm{~cm}$.

En cuanto a los nervios, los más afectados y donde se produjeron las principales articulaciones fueron el N3.4, N4.5, N5.6 y N6.7. Todos los nervios siguen la misma pauta de deformación, con un descenso del extremo superior de hasta $6 \mathrm{~cm}$, y un desplazamiento en sentido horizontal de hasta $13 \mathrm{~cm}$ hacia el exterior en el caso más desfavorable del N5.6. Se descartó la posibilidad de movimientos en el sistema de contrarresto dada la naturaleza de las lesiones y que no se observaron variaciones en las estructuras colindantes.

Se observa que el casquete S6, junto con el nervio N5.6, son las secciones más deformadas, y que coinciden con una discontinuidad de la cúpula debido a la existencia de una apertura para acceder al trasdós.

\section{MODELIZACIÓN}

Las aproximaciones numéricas tienen un doble objetivo: por una parte determinar la capacidad resistente de la cúpula para soportar el peso de la linterna, y por otra, evaluar cualitativamente el comportamiento de la cúpula sometida a diferentes solicitaciones, según su geometría. No es objeto de este estudio determinar el estado real de la estructura ni las características mecánicas de la fábrica.

La estabilidad de la cúpula es analizada por estática gráfica desde el enfoque del equilibrio (4) (5). El objetivo no es encontrar el estado real de la estructura, ya que éste puede verse alterado por infinidad de variables. Según el teorema de la seguridad, si podemos encontrar una sola línea de presiones contenida dentro de la masa de la fábrica, la estructura será estable.

El estudio del comportamiento general de la cúpula se realiza por MEF, ya que permite considerar globalmente y en tres dimensiones la totalidad de la cáscara, dada su geometría y configuración constructiva (6).

\subsection{Modelo, sistema constructivo y materiales}

A partir del modelo 3D obtenido por fotogrametría se concreta un segundo modelo sin deformar para estudiar el comporta- 
miento de la cúpula según las condiciones geométricas previas a la rotura (Figura 10). Se toman como referencia las secciones menos deformadas para deducir la geometría original.

La planta se construye a partir de los dos ejes perpendiculares (455 y $365 \mathrm{~cm}$ ), trazando dos arcos de circunferencia de $180 \mathrm{~cm}$ de radio cuyo centro se sitúa a $50 \mathrm{~cm}$ respecto el punto medio del eje longitudinal. Éstos se unen mediante dos arcos de radio $450 \mathrm{~cm}$ cuyo centro se sitúa a $270 \mathrm{~cm}$ del centro del eje transversal. La sección de la cúpula se desarrolla a partir de un arco de circunferencia con un radio que oscila entre 160 y $170 \mathrm{~cm}$, cuyo centro se sitúa a $30 \mathrm{~cm}$ por encima de la imposta. El óculo se traza a partir de dos arcos de circunferencia de $56 \mathrm{~cm}$, cuyos centros se separan apenas $15 \mathrm{~cm}$ sobre el eje longitudinal.

Teniendo en cuenta el escaso grosor de la cáscara, la técnica constructiva y su geometría con doble curvatura, podemos situar el tipo constructivo en el de bóveda tabicada (7) (8). Esta tipología se comporta de forma análoga a las bóvedas de fábrica, que transmiten empujes y tienen una resistencia baja o nula a esfuerzos de tracción.

A pesar de la esbeltez de la cáscara (51 en el eje mayor y 41 en el menor), estos valores no dejan de ser habituales en este tipo de construcción. Cabe destacar la singular proporción del peso de la linterna en relación al peso de la cúpula. Generalmente, la linterna supone entre el $5 \%$ y el $10 \%$ del peso global (9), pero en este caso representa el $65 \%$ del total. Se puede deducir de este hecho la importancia del entrevigado en la estabilidad del conjunto.

Para la caracterización de las cargas se han considerado los siguientes datos obtenidos

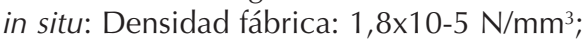
Carga cubierta: $1,5 \times 10-3 \mathrm{~N} / \mathrm{mm}^{2}$; Peso linterna: $70 \mathrm{kN}$. Complementariamente, se ensayaron en laboratorio tres probetas extraídas durante la reparación para determinar la resistencia a compresión del material. La resistencia media obtenida es de $10,1 \mathrm{~N} / \mathrm{mm}^{2}$, siendo el valor más bajo de las tres $8,7 \mathrm{~N} / \mathrm{mm}^{2}$ y el mayor de $11,84 \mathrm{~N} / \mathrm{mm}^{2}$.

Debido a la configuración constructiva y al deteriorado estado de la estructura es complicado determinar con seguridad la interacción linterna/entrevigado/cúpula en cuanto a la distribución de esfuerzos que asumen los elementos portantes. De esta forma, a partir de los datos conocidos anteriormente citados, los estudios se realizan considerando distintas hipótesis de carga aplicadas directamente en el tambor.
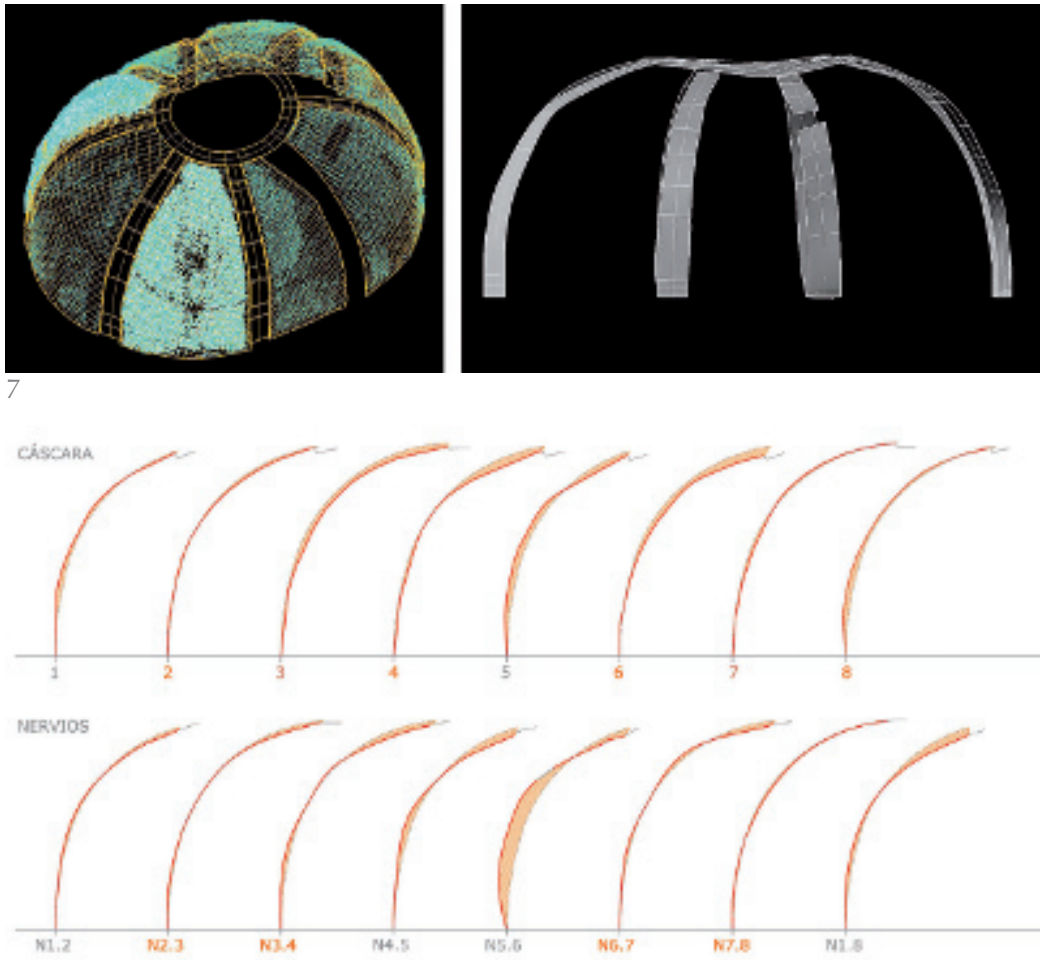

8
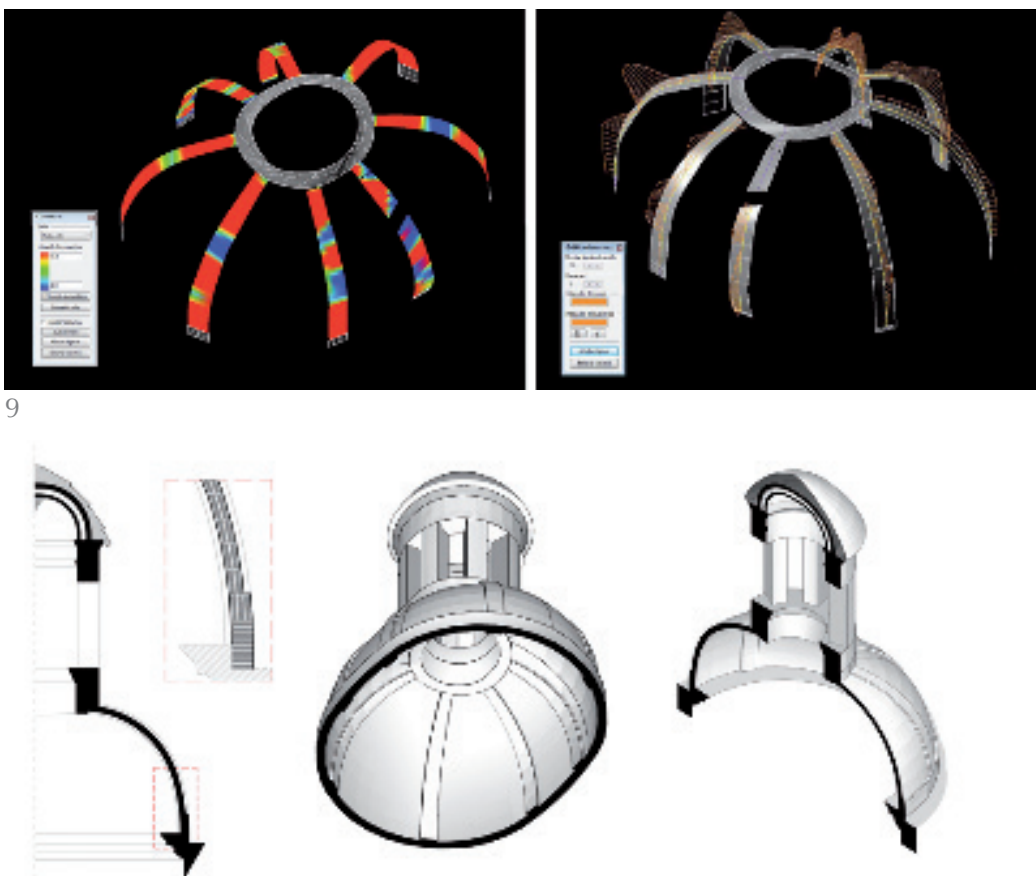

10

A tal efecto se divide su sección en 4 áreas, en función de la situación de las vigas, sobre las que se consideran diferentes hipótesis de carga según la parte asumida por las vigas.

\subsection{Análisis de la estabilidad mediante estática gráfica}

El estudio se realiza en el marco teórico del análisis límite, que considera la obra de fábrica como un material que no soporta tracciones, tiene infinita resistencia compresión y el deslizamiento entre piezas es
7. Levantamiento fotogramétrico 20 de mayo 2011

8. Estudio comparativo de las deformaciones.

9. Estudio de resultados de curvatura y deformaciones.

10. Modelo 3D. 
11. Descenso de cargas S7 $(\mathrm{H} 1 \mathrm{PP})+\mathrm{S} 5(\mathrm{H} 2$ 25\%)

12. Descenso de cargas N6.7 $(\mathrm{H} 2$ 25\%) + N5.6 (H5 100\%).
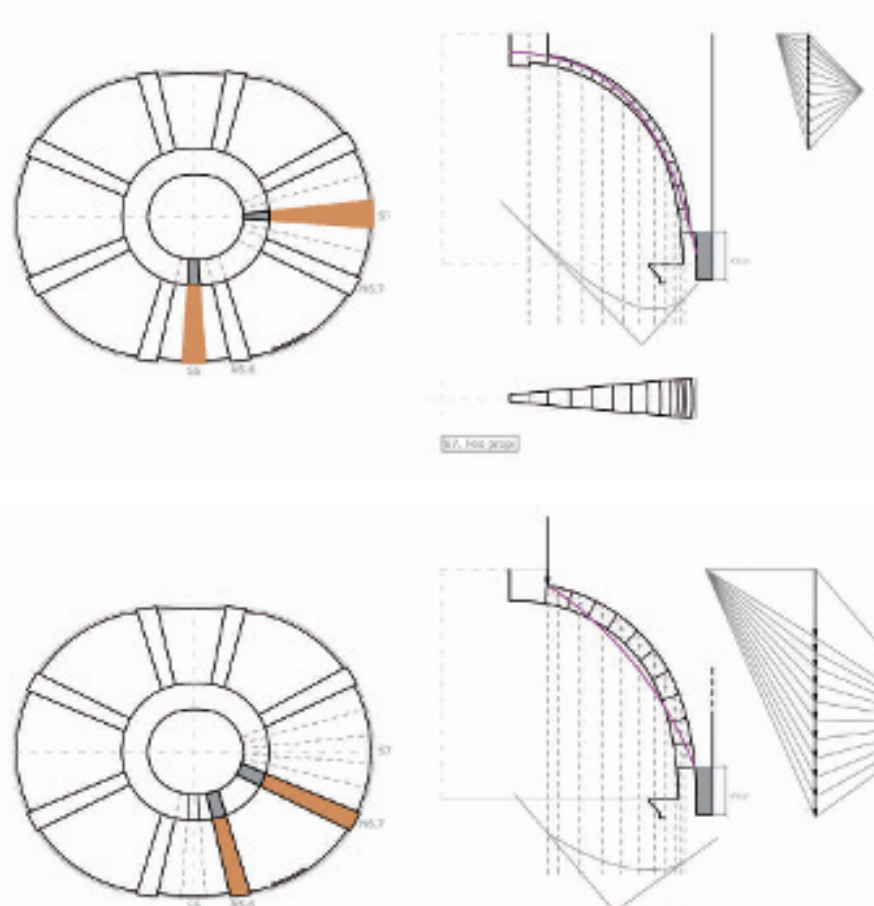

imposible. Se aplica la técnica de los cortes para estudiar la capacidad resistente de la cúpula sometida a cargas gravitatorias.

La cúpula se desarrolla a partir de 2 ejes de simetría axiales. De esta forma se estudian dos secciones características de cáscara que se corresponden con los ejes principales, así como los nervios situados entre ambos casquetes.

Inicialmente la cúpula no disponía de elementos de relleno, y la descarga de la linterna se confiaba a la estructura de madera. No obstante, tras el bombardeo de 1810, y con la reparación de esta zona, existía un cierto relleno del trasdós de la cúpula. Esta circunstancia se va a tener en cuenta en el análisis, ya que de otra forma es imposible definir una línea de empuje contenida en el grosor de la bóveda.

El reparto de cargas en el tambor no es simétrico y el relleno del trasdós tiene una altura variable. De esta forma, se analizan a continuación las condiciones de equilibrio de las secciones S5 y S7, y los nervios N5.6 y N6.7 según diferentes hipótesis de carga y relleno.

S7: para una altura de relleno del trasdós de $45 \mathrm{~cm}$ el casquete es estable sometido solo a peso propio. La hipótesis de $25 \%$ del peso de la linterna ya no presenta una solución estable (Figura 11).

S5: para una altura de relleno del trasdós de $45 \mathrm{~cm}$ el casquete es estable sometido solo a peso propio, pero la hipótesis del 25\% ya no da una solución contenida en la sección. Considerando una altura de relleno de $100 \mathrm{~cm}$, es posible encontrar una línea de presiones contenida en la sección para el $25 \%$ del peso, pero no aguantaría más (Figura 11).

N6.7: para un relleno del trasdós de $45 \mathrm{~cm}$ es posible encontrar una solución estable sometida a peso propio, pero no considerando el $25 \%$ del peso de la linterna (Figura 12).

N5.6: para un relleno del trasdós de $45 \mathrm{~cm}$ es posible encontrar una solución estable casi hasta el $50 \%$ de la carga de la linterna. Con un relleno de $100 \mathrm{~cm}$ puede soportar la totalidad del peso correspondiente de la linterna (Figura 12).

Según los resultados, la cúpula sometida solo a peso propio es estable, pero tiene una capacidad muy limitada para resistir el peso de la linterna. De esta forma, se comprueba que la colaboración con el entrevigado de madera es imprescindible para garantizar la estabilidad del conjunto. Así, un incremento de la carga asumida por la cúpula provoca la formación de rótulas y la deformación del casquete.

\subsection{Modelización mediante FEM}

El cálculo por elementos finitos se realiza con el software de libre distribución Salome-Meca 6.3. A partir del modelo 3D se
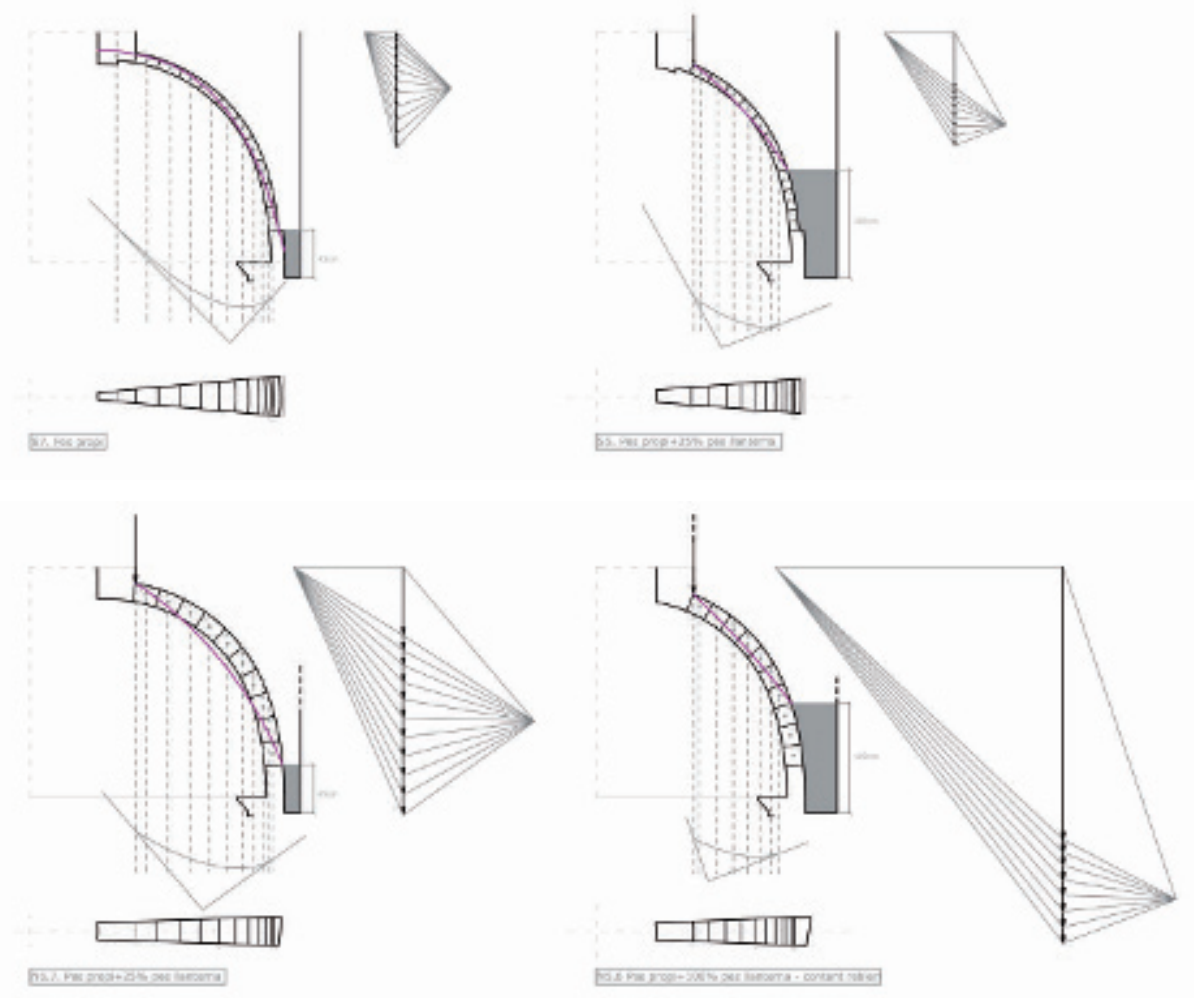
define un mallado de sólidos (Figura 13) para evaluar tensiones y desplazamientos de la fábrica considerando solo las acciones gravitatorias del peso propio. Se realizan diversos cálculos elásticos basados en un modelo no lineal con el objetivo de analizar cualitativamente el comportamiento de la cúpula en los estados iniciales del proceso.

Siguiendo trabajos anteriores (11), se asume un espacio de tensiones anisotrópico $\sigma$ y un espacio conjugado de deformaciones $\varepsilon, \Psi_{0}(\varepsilon)$ es la función inicial elástica de la energía almacenada del material. Se trata de una función convexa tal que: [1].

$$
\Psi_{0}(\varepsilon)=\frac{1}{2} \varepsilon: C: \varepsilon \geq 0
$$

Donde $C$ denota el tensor constitutivo habitual de cuarto orden, isotrópico lineal elástico. Se asume la forma de la energía libre potencial: [2].

$$
\Psi(\varepsilon, r)=(1-d(r)) \Psi_{0}
$$

Donde habitualmente $r$ es una variable interna que puede ser representada como el umbral de daño, $d(r)$ es una función escalar de la variable inercial tal que $0 \leq d(r) \leq 1$. Por tanto, la ecuación constitutiva que se obtiene es: [3].

$$
\sigma=\frac{\partial \Psi}{\partial \varepsilon}=(1-d(r)) C: \varepsilon
$$

Finalmente, se usa la ecuación constitutiva en términos del campo de tensiones en el espacio isotrópico, tenemos: [4].

$$
\sigma=\left(A^{\sigma}\right)^{-1}: A^{\sigma}: \sigma
$$

Donde $A^{\sigma}$ es el tensor de transformación de tensiones relativo a los espacios canónicos y reales. Finalmente, para el cálculo se realiza una simplificación considerando un único material homogéneo, con unas características mecánicas habituales: Módulo de Young: $5000 \mathrm{~N} / \mathrm{mm}^{2}$; Coef. de Poisson: 0,15 (12) (13). El resto de propiedades de material se extraen de (14) para el test uniaxial de tensión/compresión, y $d(r)$ es una función constante para cada simulación.

Se plantean diferentes modelos para tantear el comportamiento de la cúpula en función del reparto de las cargas en el cuello de la linterna. En las diferentes hipótesis siempre se considera que las cargas gravitatorias se reparten entre la estructura de madera y la cúpula ya que, tal y como se ha comprobado, ésta última no sería capaz de soportar la totalidad de la carga.

Se realizan tres simulaciones según diferentes estados tensionales: en primer lugar se analiza el comportamiento ideal en un estado de carga uniforme, para después

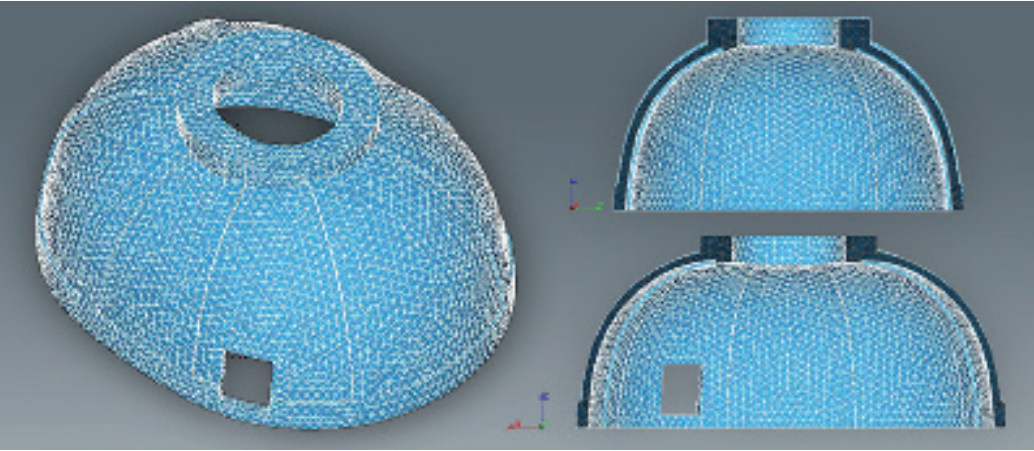

13
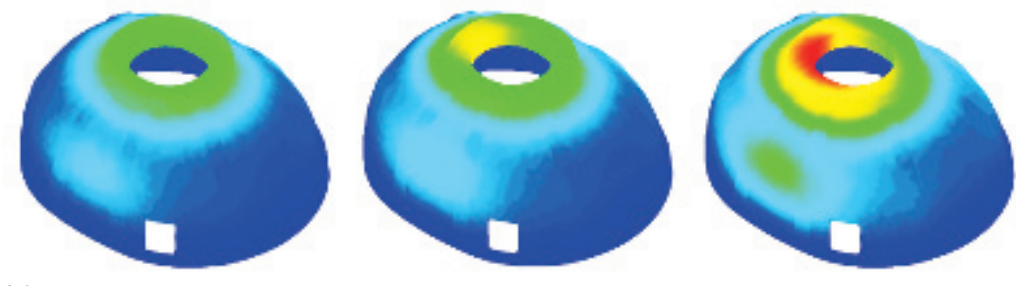

14
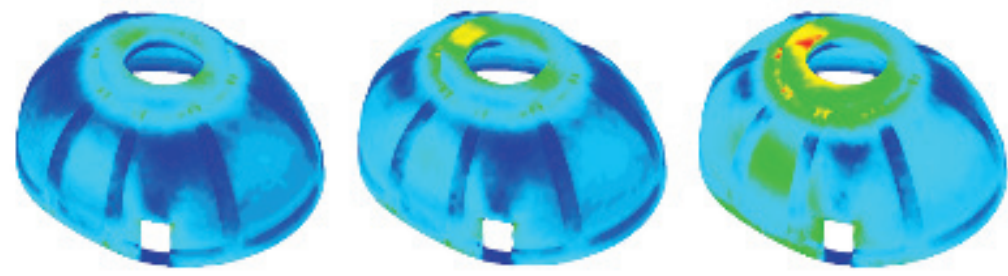

simular el reparto de los pesos de cubierta y linterna en función de los apoyos del entrevigado. Finalmente se considera la carga asimétrica que provocó la rotura.

13. Malla 3D.

14. Modelos de deformaciones. 15. Modelos de tensiones

Modelo 1 (Figuras 14 y 15), comportamiento ideal con carga uniforme: las mayores deformaciones aparecen, por una parte, alrededor del óculo, y por otra, en los casquetes con mayor pendiente - eje menor de simetría- especialmente a $1 / 2$ de su altura. Las tensiones aparecen con mayor intensidad en los extremos de los casquetes, y también a media altura en los ejes axiales, especialmente en los de mayor pendiente. En los nervios aparecerán mayores tensiones en los extremos del extradós y en la parte central del intradós.

Modelo 2 (Figuras 14 y 15), comportamiento teórico inicial con cargas diferenciales: se considera la misma tensión que en el Modelo 1 para Q2 y Q4, Q1 soporta una carga ligeramente inferior y Q3 soporta una carga superior, equivalente al peso correspondiente de la linterna y cubierta. Se observa como la carga diferencial no supone variaciones significativas en el comportamiento general de la cúpula respecto el Modelo 1, más allá del incremento de tensiones y deformaciones en el lado más cargado.

Modelo 3 (Figuras 14 y 15), comportamiento con carga asimétrica: partiendo del Modelo 2, se aplica en Q4 el incremento de 
16. Mecanismo de colapso

17. Comparativa roturas y modelos deformaciones / tensiones. carga correspondiente al peso de linterna y cubierta, antes soportado por la viga. Como es lógico, esto provoca un incremento tanto de las tensiones como de las deformaciones en el lado correspondiente de la cúpula, siguiendo el comportamiento explicado en los modelos anteriores. Las tensiones se concentran en las superficies al rededor del óculo y en la base, así como en el tercio superior del intradós de los nervios correspondientes.

\section{RELACIÓN DE LOS RESULTADOS CON LA ROTURA REAL}

Los desplazamientos causados por la carga asimétrica en el tambor provocan un proceso de acomodación de la fábrica en el que se redistribuyen progresivamente las tensiones, modificando la línea de presiones. La cáscara rompe y se deforma para adaptarse a las nuevas solicitaciones y, en el último estadio, se forman las rótulas que pondrán en marcha el mecanismo cinemático de colapso.

La formación de rótulas (Figura 16) aparece a niveles distintos para el caso de nervios y cáscara, ya que no tienen la misma rigidez; primero rompen radialmente los nervios,
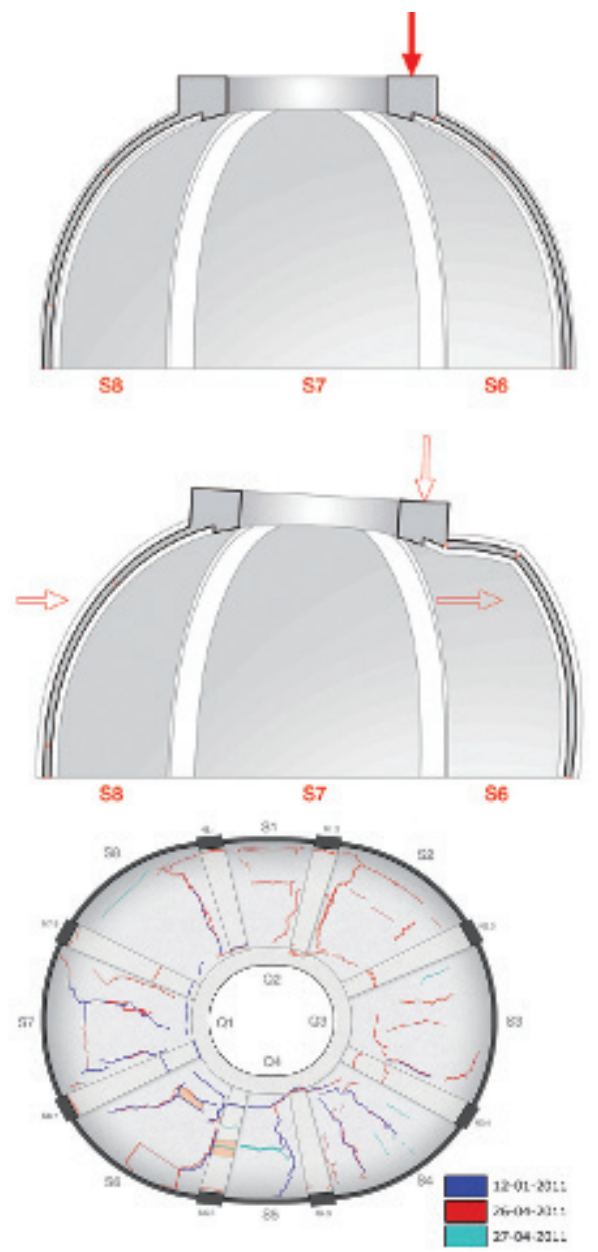

en la parte superior alrededor del óculo, a la vez que aparecen grietas meridianas en los casquetes. En un segundo estadio, rompe la cáscara a media altura y aparecen más grietas meridianas, especialmente cerca de los nervios.

Se ha comprobado que la cúpula por sí sola difícilmente podría soportar el peso de la linterna. La existencia de material de relleno en el trasdós de cúpulas y arcos siempre tiene un papel clave en su estabilidad. En este caso, el relleno es heterogéneo y con una altura muy variable, de forma que el entrevigado debía asumir una parte importante del peso de la linterna.

Según los resultados de la modelización (Figura 17), las tensiones tienden a ser bajas, con valores entre 0,15 y $0,3 \mathrm{~N} / \mathrm{mm}^{2}$ en los puntos más desfavorables. Según los ensayos realizados, estos valores están por debajo de la tensión de rotura del material. Por otra parte es posible relacionar la formación de grietas con las zonas donde hay mayor gradiente tensional, y no se producirán necesariamente en las partes más solicitadas.

Por otra parte, en el caso de cúpulas esféricas, la distribución de tensiones en la superficie del casquete tiende a ser uniforme. Esto no es así en geometrías ovales, donde los esfuerzos varían según las diferentes pendientes y radios de curvatura. Este hecho se ve acentuado por la existencia de los 8 nervios meridianos, que determinan zonas con diferente rigidez. Tal y como muestran los modelos, el reparto de

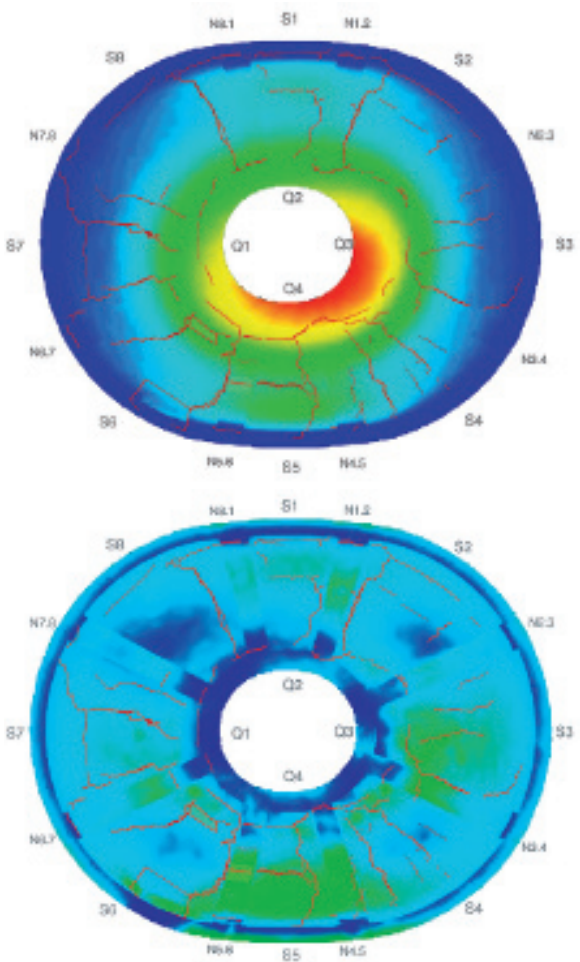


tensiones no es homogéneo, aún cuando se considera una carga uniforme en el tambor.

El casquete más afectado por la fisuración fue el S6, que disponía de un pequeño orificio y no daba continuidad a la estructura geométrica de la cúpula. Éste, junto con el nervio N5.6, fueron los que presentaron mayores desplazamientos y en consecuencia los que más dañados estaban, a pesar de que las tensiones no eran especialmente altas.

\section{CONCLUSIONES}

El estudio concluye que los desplazamientos causados por la aplicación de una carga asimétrica en el óculo de la cúpula provocan un proceso de acomodación de la geometría de la fábrica, modificando su equilibrio inicial. Así, debido al escaso grosor de la cáscara, la cúpula cede bajo el peso de la linterna y se produce una redistribución en el reparto de esfuerzos. La línea de presiones varía hasta que, en el último estadio, se forman las rótulas que pondrán en marcha el mecanismo cinemático de colapso. De esta forma, la fábrica rompe a causa de las tensiones provocadas por las variaciones geométricas y no como consecuencia directa de la aplicación de una carga. A demás, se intuye una relación entre la formación de grietas y las zonas con mayor gradiente tensional, que no necesariamente deben coincidir con las secciones más solicitadas.
Inicialmente, se observa la aparición de las fisuras en el intradós de la parte superior cercana al óculo. La fábrica de la cúpula tiende a romper en las zonas próximas a los nervios y al cinturón del óculo, debido a las diferencias de rigidez. En un segundo estadio, se visualiza en el intradós la rótula inferior (con su apertura en el extradós). De esta manera concurren las mismas hipótesis que en la rótula inicial, a pesar de que el diferencial de tensión respecto a los puntos superiores de la cúpula sea menor.

La apariencia, secuencia y sintomatología de las fisuras observadas, cumple con la teoría de rotura de cúpulas dentro del análisis límite. Coincide además con las modelizaciones gráficas donde los puntos críticos de las líneas de empuje tienden a formar las articulaciones.

La cúpula finalmente no colapsó gracias a que se frenó el proceso cinemático mediante el acodalamiento de la linterna. La existencia del relleno del trasdós, con aquellos escombros del bombardeo del 30 de diciembre de 1810, evitó la completa formación de la grieta meridiana inferior, entre los nervios N5.6 y N6.7. Esta situación evitó el giro hacia el extradós del casquete y el colapso de la cúpula. Finalmente la cúpula oval se reparó sin ser desmontada, rigidizando la bóveda de fábrica mediante la técnica de esparto y yeso.

\section{REFERENCIAS}

(1) Almuni, V., Lluís, J. (2000). Sancta Maria Dertosae. Catedral de Tortosa. Guia histórica i descriptiva. Tortosa, Capítol Catedral de Tortosa - Bisbat de Tortosa.

(2) Huerta S. (2004). Arcos, bóvedas y cúpulas de fábrica. Madrid, Instituto Juan de Herrera.

(3) Heyman J. (1995). The stone skeleton: structural engineering of masonry architecture. Cambridge, Cambridge University Press.

(4) Huerta, S. (2001). Mechanics of masonry vaults: the equilibrium approach. Proc. of the 1st Int. Cong. on Structural Analysis of Historical constructions, pp. 47-70, Guimaraes, eds. P.B.Lourenço \& P.Roca.

(5) Block, P.; DeJong, M.; Ochsendorf, J. (2006). As Hangs the Flexible Line: equilibrium of Masonry Arches. Nexus Network Journal, 8(2): 13-24.

(6) Chiorino, M.A.; Spadafora, A.; Calderini, C.; Lagomarsino, S. (2008). Modeling Strategies for the World's Largest Elliptical dome at Vicoforte. Internationa Journal of Architectural Heritage: Conservation, Analysis, and Restoration, 2(3): 274-303.

(7) Moya, L. (2000). Bóvedas tabicadas. Dirección General de la Vivienda, la Arquitectura y el Urbanismo. $2^{\mathrm{a}}$ ed. Madrid, Ministerio de Fomento. Centro de Publicaciones.

(8) Huerta, S. (2006). Escritos sobre la construcción cohesiva y su función en la arquitectura. Rafael Guastavino. Madrid, Instituto Juan de Herrera.

(9) Huerta, S.; López, G. (2001). Estudios estructurales previos a la restauración de la iglesia de Santo Tomás de Villanueva ("La Mantería") de Zaragoza. Instituto de Patrimonio Histórico Español, Madrid.

(10) Wolfe, W. S. (1921). Graphical Analysis: A textbook on graphic statics. McGraw-Hill Book Co. New York.

(11) Simó, J.C., Ju, J.W. (1987). Strain- and stress-based continuum damage models, I. Formulation. International Journal Solids Structures 23: 821-840.

(12) Lourenço, O.B. (1996). Computational Strategies for Masonry Structures. Delft University Press. Delft.

(13) Alonso, A., Gomis, J., Moreno, J., Llopis, V. (2011). Arquitectura religiosa: anàlisis constructivo y estructural. Actas del Sétpimo Congreso Nacional de História de la Construcción. pp. 55-64.

(14) Pelà, L., Cervera, M., Roca, P. (2011). Continuum damage model for orthotropic materials: Application to masonry. Comput. Methods Appl. Mech. Engrg., 200: 917-930. 